\title{
CAUSA VERSUS PREDIÇÃO: HISTÓRIA DE BANHOS EM RIOS COMO FATOR DE RISCO E PREDITOR DA INFECÇÃO PELO SCHISTOSOMA MANSONI*
}

\author{
Mauricio L. Barreto**
}

\begin{abstract}
BARRETO, M. L. Causa versus predição: história de banhos em rios como fator de risco e preditor da infecção pelo Schistosoma mansoni. Rev. Saúde publ., S. Paulo, 21:305-9, 1987.

RESUMO: Através da análise das relações existentes entre as medidas de risco e as medidas de eficiência de testes de diagnóstico ou triagem, são discutidos os problemas e limitações concernentes ao uso de fatores de risco na predição da distribuição de efeitos (infecção, doença, cura) em uma população. Para ilustrar o tema, foi utilizada a associação, em crianças, entre a questão "você já tomou banhos em rios?", muito utilizada em anamneses clínicas ensinadas em diversas escolas médicas do Brasil, e a infecção pelo Schistosoma mansoni. A história de banhos em rios, enquanto um importante fator de risco, mostrou-se mau preditor da ocorrência da infecção esquistossomótica. A análise demonstrou que este achado é, em grande parte, devido a baixa frequiência de história negativa de banhos em rios. Porém, a possibilidade da utilização de fatores de risco isoladamente ou agrupados, com o objetivo de predição de efeitos, é possível e desejável. Apesar de pouco explorado nos seus aspectos práticos ou conceituais, esta área de conhecimento representa um importante ponto de convergência entre a epidemiologia das causas e a epidemiologia das intervenções.
\end{abstract}

UNITERMOS: Métodos epidemiológicos. Risco. Previsóes. Efetividade. Esquistossomose mansônica, prevenção e controle.

\section{INTRODUÇÃO}

A tomada de decisões é parte rotineira da prática de médicos e profissionais de saúde pública. A indicação de um exame com objetivo diagnóstico ou triagem para um paciente ou uma população, a indicação de uma medida terapêutica ou profilática, são exemplos da constante necessidade desses profissionais em decidir entre duas ou mais opções. Para aqueles envolvidos neste tipo de prática, o surgimento de novos conhecimentos, que melhorem o grau de confiabilidade envolvido em suas decisões, é de máxima importância.

Como conseqüência da intensificação do uso do método epidemiológico nas investigaçōes em saúde, um número cada vez maior de eventos mórbidos tem tido a sua ocorrência associada a fatores de risco específicos. Em geral, a demonstração da existência da associação entre um atributo, o qual pode ser um fator de risco, uma intervenção ou um teste diagnóstico, e um efeito, o qual pode ser uma infecção, uma doença ou uma cura, tem implicações importantes. Dois grandes grupos de ações podem ser gerados do conhecimento obtido: a) proteger ou expor (na dependência da qualidade do efeito ou do atributo) a população à ação do atributo; b) utilizar a presença ou ausência do atributo como indicativo da presença ou ausência do efeito. $O$ item $a$ concerne às investigações cujo objetivo seja estudar associações de risco ou eficácia de intervenções. $O$ item $b$ diz respeito às investigações dirigidas à avaliação de testes de diagnóstico ou triagem.

A idéia de teste de diagnóstico ou triagem é bastante ampla para incluir uma série de técnicas, que não se limitam aos clássicos exames clínicos ou laboratoriais. Por exemplo, um questionário ou mesmo uma simples questão, em função de uma série de características, entre as quais se inclui o seu poder discriminante, pode ser considerada como tal. Um fator de risco, principalmente quando o risco relativo assume altos valores, pode vir a ter função similar 1 .

* Pesquisa realizada com apoio do "UNDP/World Bank/WHO Special Programme for Research and Training in Tropical Diseases".

* Departamento de Medicina Preventiva, Universidade Federal da Bahia. Vale do Canela - $40000-$ Salvador, BA - Brasil. Bolsista de Doutorado do CNPq no "Evaluation and Planning Centre for Health Care" da "London School of Hygiene and Tropical Medicine". 
$E$ antigo o conhecimento da relação entre - contato com coleçōes hídricas e a ocorrência de esquistossomose mansônica. A questão, "você já tomou banho de rio?", faz parte das anamneses ensinadas em diversas escolas médicas do Brasil. Trabalhos epidemiológicos recentes têm quantificado o papel de banhos em rios como fator de risco nessa endemia e, ao menos em crianças, têm mostrado uma associação de grande magnitude (altos riscos relativos $)^{7,8,11}$. Entretanto, nada tem sido feito para avaliar a utilidade desta questão como elemento de medida da probabilidade do paciente estar ou não infectado, justificando, assim, a sua inserção na anamnese clínica.

Através da análise das relações existentes entre a história de banhos em rios (atributo) e a infecção pelo Schistosoma mansoni (efeito), buscaremos explorar as limitações do uso de um atributo, causalmente associado a um efeito, como elemento preditor da ocorrência deste mesmo efeito. Nosso estudo ficará restrito à análise das inter-relações entre o risco relativo, as medidas de eficiência de um teste $e$ as respectivas frequiências do efeito $e$ do atributo.

\section{POPULAÇÃO E METODOS}

Como parte de um programa de investigação sobre fatores associados à ocorrência da esquistossomose mansônica em meio urbano, um inquérito epidemiológico foi desenvolvido na cidade de Santo Antonio de Jesus (Bahia). A cidade situa-se a $100 \mathrm{~km}$ a oeste de Salvador (capital de Estado). Sua população em 1984 foi estimada em 45.000 habitantes, apresentando taxas de crescimento populacional muito acima da média do Estado, fato decorrente do forte componente imigratório. Até o momento do inquérito, a cidade não tinha sido alvo de nenhuma ação programada de controle dessa endemia.

Uma das amostras do estudo constou de todas as crianças nascidas entre $01 / 01 / 1970$ e $31 / 12 / 1971$ morando na cidade, no momento do inquérito. $O$ estudo de campo foi desenvolvido no período de agosto a outubro de 1984. Essas crianças tinham, portanto, no referido período, entre 12 e 14 anos de idade. Para encontrar este grupo populacional específico, todos os domicílios da cidade foram visitados. Para cada criança encontrada, aplicou-se um questionário sobre as condições sociais e econômicas da família e um outro questionário dirigido à própria criança. Neste último, constava, entre outras, a questão: "Você já tomou banho em rios?", com duas possibilidades de resposta: sim ou não.

\section{Exame Parasitológico de Fezes}

Para cada criança distribuiu-se um pequeno vaso identificado, o qual era coletado, com a amostra de fezes, em uma data previamente definida. $O$ exame parasitológico foi feito pela técnica quantitativa de Kato modificada por Katz e col. ${ }^{9}$. Para cada amostra de fezes, duas lâminas foram preparadas e examinadas. A carga parasitária individual foi definida como a média da contagem das duas lâminas e expresso em número de ovos por grama de fezes. $\mathrm{Na}$ presente análise, consideraremos três níveis de positividade: positivos em geral (1 ou mais ovos/g de fezes), positivos com mais de 100 ovos/g de fezes e positivos com mais de 400 ovos/g de fezes. Esta estratificação é importante em virtude da forte relação existente entre carga parasitária, medida pelo número de ovos nas fezes, e a gravidade da doença $a^{2,3,10,13}$.

\section{Métodos Estatísticos}

A análise foi desenvolvida através do estudo das inter-relaçōes entre os diversos parâmetros extraídos de tabelas do tipo $2 \times 2$. A Tabela 1 apresenta o esquema básico de uma tabela deste tipo. Suporemos que tal tabela é proveniente de uma população não selecionada, quer seja em função do atributo ou do efeito. Portanto, isto significa que não houve definição prévia dos seus valores marginais.

\section{TABELA 1}

Estrutura básica de uma tabela $2 \times 2$ relacionando 0 atributo (A) ao efeito (E).

\begin{tabular}{cccc}
\hline & $\mathbf{E}$ & $\mathbf{E}$ & Total \\
\hline $\mathbf{A}$ & $\mathbf{a}$ & $\mathbf{b}$ & $\mathbf{a}+\mathbf{b}$ \\
$\mathbf{A}$ & $\mathbf{c}$ & $\mathbf{d}$ & $\mathrm{c}+\mathbf{d}$ \\
Total & $\mathbf{a}+\mathbf{c}$ & $\mathbf{b}+\mathbf{d}$ & $\mathbf{N}$ \\
\hline
\end{tabular}

A seguir estão descritos os parâmetros que serão utilizados ${ }^{4,6}$ :

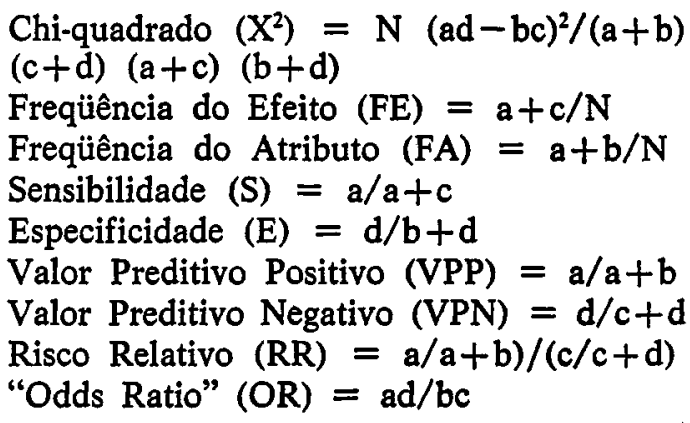

\section{RESULTADOS}

Um total de 1.765 crianças, nascidas no período compreendido entre $1 / 1 / 1970$ e $31 / 12 / 1971$, foram encontradas morando na 
cidade. Deste total, 1.700 forneceram informaçōes completas para o presente estudo.

A análise das Tabelas 2,3 e 4 , sumarizadas na Tabela 5, mostram forte associação entre a história de banhos em rios e estar infectado pelo $S$. mansoni $\left(\mathrm{X}^{2}=34,1,19,4\right.$ e 11,4 , respectivamente, com $\mathrm{p}<0,001 \mathrm{em}$ todos os três casos). Os valores dos "Odds Ratios" $(4,8,8,7 \mathrm{e}$ indeterminado, respectivamente) confirmam sua importância como fator de risco na infecção esquistossomótica, principalmente para o grupo com altas cargas parasitárias. Se, por outro lado, procurarmos observar a utilidade deste atributo em discriminar infectados de não-infectados, teremos os seguintes resultados: altos níveis de sensibilidade $(0,975,0,986$ e 1,00 , respectivamente) e altos VPNs $(0,908,0,977$ e 1,00 , respectivamente), porém, baixas especificidades $(0,109$ em todos os casos) e baixos VPPs $(0,330,0,169$ e 0,074 , respectivamente). A prevalência da infecção (FE) e a frequiência de banhos em rios (FA) encontradas foram respectivamente: 0,310 e 0,917 para infectados em geral, 0,156 e 0,905 para infectados excretando mais de 100 ovos/g de fezes e 0,074 e 0,899 para aqueles excretando mais de 400 ovos/g de fezes (Tabela 5).

TABELA 2

Associação de história de banhos (HB) em rios (atributo) e infecção (I) pelo $S$. mansoni (efeito) em crianças de 12-14 anos de idade. Santo Antônio de Jesus, Bahia, Brasil, 1984.

\begin{tabular}{cccc}
\hline & I (+) & I (-) & Total \\
\hline HB (+) & 514 & 1045 & 1559 \\
HB (+) & 13 & 128 & 141 \\
Total & 527 & 1173 & 1700 \\
\hline
\end{tabular}

TABELA 3

Associação entre história de banhos (HB) em rios e infecção (I), com mais de 100 ovos/g de fezes, pelo $S$. mansoni. Santo Antônio de Jesus, Bahia, Brasil, 1984.

\begin{tabular}{cccc}
\hline & I (+) & I (-) & Total \\
\hline HB (+) & 213 & 1045 & 1258 \\
HB ( $)$ ) & 3 & 128 & 131 \\
Total & 216 & 1173 & 1267 \\
\hline
\end{tabular}

TABELA 4

Associação entre história de banhos em rios (HB) e infecção (I), com mais de 400 ovos/g de fezes, pelo S. mansoni. Santo Antônio de Jesus, Bahia, Brasil, 1984.

\begin{tabular}{cccc}
\hline & $I(+)$ & $I(-)$ & Total \\
\hline HB $(+)$ & 94 & 1045 & 1139 \\
HB $(-)$ & 0 & 128 & 128 \\
Total & 94 & 1173 & 1267 \\
\hline
\end{tabular}

TABELA 5

Sumário dos parâmetros extraídos das Tabelas 2,3 e 4

\begin{tabular}{lccc}
\hline & Tab. 2 & Tab. 3 & Tab. 4 \\
\hline FE & 0,310 & 0,156 & 0,074 \\
FA & 0,917 & 0,905 & 0,899 \\
X $^{2}$ & $34,1^{*}$ & $19,4^{*}$ & $11,4^{*}$ \\
OR & 4,8 & 8,7 & indeterm. \\
& $(2,6-8,3)+$ & $(2,7-23,5)+$ & \\
E & 0,109 & 0,109 & 0,109 \\
VPP & 0,330 & 0,169 & 0,083 \\
VPN & 0,908 & 0,977 & 1,000 \\
\hline
\end{tabular}

$* \mathrm{p}<0,001$

$+95 \%$ Limites de Confiança

FE - Freqüência de efeito

FA - Frequiência de atributo

$\mathrm{X}^{2}$ - Quiquadrado

OR - "Odds Ratio"

E - Especificidade

VPP - Valor preditivo positivo

VPN - Valor preditivo negativo

\section{DISCUSSĀO}

Apesar do desenvolvimento dos instrumentais analíticos em epidemiologia e bioestatística, as tabelas $2 \times 2$ continuam sendo peças fundamentais nos estudos epidemiológicos e nos estudos de avaliação dos testes diagnósticos. Entretanto, a organização dessas tabelas, bem como sua exploração, estão na dependência do objetivo com que são construídas. Nos estudos caso-controle ou de avaliação de testes, predefine-se um grupo que tem o efeito, comparando-o com um grupo que não o tem, sendo o objetivo, em ambos os casos, observar a distribuição do atributo, ainda que por diferentes caminhos. Em acordo com a notação aqui utilizada, as marginais $a+c$ e $b+d$ têm seus valores definidos no desenho destes estudos. Nos estudos de coortes, define-se um grupo que possui o atributo, comparando-o a um grupo similar, porém no qual o atributo está ausente. $\mathrm{Na}$ notação aqui utilizada, podemos dizer que os valores das marginais $a+b$ e $c+d$ são previamente definidas.

Nos estudos de coortes a medida do risco é feita pelo Risco Relativo (RR), o qual é dependente das marginais do atributo. Nos estudos de caso-controle tal medida é feita pelo "Odds Ratio" (OR), o qual é, por definição (ver Metodologia), completamente independente dos valores marginais. No caso em que a frequiência do efeito $(a+c / N)$ seja muito pequena, demonstra-se que $R R \cong O R$, o que torna, em princípio, similares os resultados de estudos epidemiológicos de coorte e caso-controle 4 .

Dos parâmetros definidos como de utilidade na avaliação de um teste, veremos que dois 
deles ( $\mathrm{S}$ e E) são dependentes das marginais relacionadas ao efeito, enquanto que os dois outros (VPP e VPN) são dependentes das marginais relacionadas ao atributo. Desta observação, pode-se inferir que, para uma completa avaliação de um teste, o desejado seria a não predefinição dos valores marginais em nenhum dos dois sentidos. Em outras palavras, dever-se-ia trabalhar com populações não selecionadas. Entretanto, alternativamente, é possível conhecendo-se previamente o valor de FE, deduzir-se VPP e VPN com base nos valores de $E$ e $S^{14}$.

Em nossos resultados, encontramos uma forte associação estatística entre a história de banhos em rios e a presença de infecção esquistossomótica. Os "Odds Ratios" calculados também foram altos, o que confirma o conhecimento sobre o papel de banhos em rios como fator de risco na infecção pelo $S$. man$\operatorname{soni}^{7,8,11}$.

Ao avaliarmos a história de banhos em rios, como um fator preditor da probabilidade de um indivíduo estar ou não infectado, outros parâmetros precisam ser analisados. Dois destes parâmetros (S e VPN) assumem altos valores. A partir dos VPNs encontrados, podemos deduzir que, na população estudada, história de banhos em rios negativa associa-se com baixa probabilidade de infecção esquistossomótica em geral e faz improvável o encontro de infectados excretando mais de 400 ovos/g de fezes. Os dois outros parâmetros (E e VPP) assumem valores muito baixos. Os valores do VPP, em cada caso, são muito próximos aos valores das respectivas prevalências $(\mathrm{FE})$ $(0,330 \times 0,310,0,169 \times 0,156$ e $0,083 \times 0,074$, respectivamente). A pequena diferença entre os valores assumidos por VPP e $\mathrm{FE}^{5}$ mostra que uma história positiva de banhos em rios pouco acrescenta à probabilidade de se determinar uma infecção esquistossomótica sem o auxílio desta informação.

As inter-relações existentes entre estes diversos parâmetros podem ser melhor entendidas pela discussão de duas equações. Estas, por sua vez, podem ser facilmente deduzidas das definições apresentdas na Metodologia: a) $\mathrm{VPP}=\mathrm{SxFE} / \mathrm{FA}$ ou seja, uma alta frequiência do atributo, tal qual aconteceu em nosso exemplo, contribui para que o VPP assuma valores pequenos;

b) $R R=V P P /(1-V P N)$, a qual torna evidente que o encontro de um alto RR pode acontecer em situações em que exista um alto VPN acompanhado por um baixo VPP, tal qual verificado no caso em foco.

O problema estudado pode ser aplicado a muitas outras questões, existentes em investigações clínicas ou epidemiológicas, que tenham por objetivo predizer a distribuição do efeito com base na presença ou ausência do atributo. Mesmo considerando que estes tipos de investigações se fundamentam na associação de um grande número de informações, a avaliação isolada das questões contidas em seus instrumentos pode melhorar o grau de confiabilidade destes, bem como melhorar o conhecimento sobre os seus poderes e limitaçōes como elementos preditores das probabilidades de distribuição do efeito.

$O$ encontro de um alto RR (ou $O R$ ), enquanto uma importante medida de associação do tipo causa-efeito e como conseqüência também importante na definição de medidas preventivas, isoladamente, não é útil como medida da distribuição do efeito entre aqueles que têm ou não o atributo. Com esta afirmação, não queremos descartar a potencialidade do uso de fatores de risco, isoladamente ou agrupados, como elementos capazes de discriminar a presença ou ausência do efeito ${ }^{1}$. Porém, para que este poder discriminante seja demonstrado, necessita-se de outras medidas que não somente o RR. $O$ fato de que $O$ risco relativo e os parâmetros de eficiência de um teste possam ser extraídos de uma mesma tabela $2 \times 2$, os tornam medidas inter-relacionáveis, porém não da mesma grandeza ${ }^{12}$.

Este campo em comum entre fatores causais e fatores preditores, ainda pouco explorado nos seus elementos práticos ou conceituais, representa um importante ponto de convergência entre a epidemiologia das causas e a epidemiologia das intervenções. 
BARRETO, M. L. Cause versus prediction: the history of river-bathing as a risk factor and a predictor of infection by Schistosoma mansoni. Rev. Saúde públ., São Paulo, 21:305-9, 1987.

ABSTRACT: The analysis of the relationship between those parameters used to measure risk and those used to measure efficiency of diagnostic or screening tests is used, in this paper, to demonstrate the limitations of the use of risk factors as predictors of outcomes distribution in a population. The issue was illustrated by the study of the association, in children, of reported bathing in rivers by asking children if they bathed in rivers and the occurrence of Schistosoma mansoni infection. This question in the above context is important because it is part of the clinical anamnesis taught in several Brazilian medical schools. However, while the history of bathing in rivers proved to be a high risk factor it was a bad discriminator between infected and non-infected children. This finding is demonstrated to be mainly related to the low frequency of people with a negative history of bathing in rivers. But, in general, it was shown that the use of isolated or grouped risk factors in the prediction of outcomes is possible and desirable. The field of knowledge discussed in this paper is not yet well explored in its conceptual and practical aspects. However, it represents an important point of convergence between the epidemiology of the causes and the epidemiology of the interventions.

UNITERMS: Epidemiology methods. Risk. Forecasting. Effectiveness. Schistosomiasis mansoni, prevention and control.

\section{REFERENCIAS BIBLIOGRÁFICAS}

1. BACKETT, E. M.; DAVIES, A. M.; PETROS-BARVAZIAN, A. The risk approach in health care: with special reference to maternal and child health, including family planning. Geneva, World Health Organization, 1984. (Public Health Papers, 76).

2. BARRETO, M. L. \& LOUREIRO, S. The effect of Schistosoma mansoni infection on child morbidity in the State of Bahia (Brasil). I - Analysis at the ecological level. Rev. Inst. Med. trop. S. Paulo, 26:230-5, 1984.

3. BARRETO, M. L.; LOUREIRO, S.; MELO, A. S.; ANJOS, C. F. D. The effect of Schistosoma mansoni infection on child morbidity in the State of Bahia. II - Analysis at the individual level. Rev. Inst. Med. trop. S. Paulo, 27:167-71, 1985.

4. BRESLOW, N. E. \& DAY, N. E, Statistical methods in cancer research. I - The analysis of case-control studies. Lyon, IARC, 1980. (IARC Scientifical Publications, 32).

5. CONNEL, F. A. \& KOEPSEL, T. D. Measures of gain in certainity from a diagnostic test. Amer. J. Epidem., 121:744-53, 1985.

6. FEINSTEIN, A. R. Clinical biostatistics. XXXI On the sensitivity, specificity and discrimination of diagnostic tests. Clin. Pharmacol. Ther., 15:528-40, 1975.

7. GUIMARÃES, M. D. C.; BARROS, H. L.; KATZ, N. A clinical epidemiological study in a schistosomiasis mansoni endemic area (Tuparecê, Minas Gerais). Rev. Inst. Med. trop. S. Paulo, 27:123-31, 1985.

8. GUIMARÃES, M. D. C.; COSTA, M. F. F. L.; LIMA, R. B.; MOREIRA, M. A. Estudo cli- nico-epidemiológico da esquistossomose mansoni em escolares da Ilha, Município de Arcos, MG (Brasil), 1983. Rev. Saúde públ., S. Paulo, 19:8-17, 1985.

9. KATZ, N.; CHAVES, A.; PELLEGRINO, J. A simple device for quantitative stool thick-smear technique in schistosomiasis mansoni. Rev. Inst. Med. trop. S. Paulo, 14:397-400, 1972.

10. LEHMAN, J. S.; MOTT, K. E.; MORROW, R. H.; MUNIZ, T. M.: BOYER, M. H. The intensity and effects of infection with Schistosoma mansoni in a rural community in North-East Brazil. Amer. J. trop. Med. Hyg., 25:285-94, 1976.

11. LOUREIRO, S. Schistosomiasis mansoni in children - an epidemiological study of patterns of water exposure using path-analysis. Houston, 1978. [PhD Thesis - University of TeXas].

12. MURPHY, J. R. The relationship of relative risk and positive predictive value in $2 \times 2$ tables. Amer. J. Epidem., 117:86-9, 1983.

13. SLEIGH, A.; HOFF, R.; MOTA, E. A.; SHERLOCK, I.; MOTT, K, E.; BARRETO, M. L.; MAGUIRRE, J. H.; WELLER, T. H. Three year prospective study of the evolution of manson's shistosomiasis in northeast Brazil. Lancet, 2:63-6, 1985.

14. VECCHIO, T. J. Predictive value of a single diagnostic test in unselected population. New Engl. J. Med., 274:1171-3, 1966.

Recebido para publicação em 11/12/1985 Reapresentado em 13/5/1987 Aprovado para publicação em 15/5/1987 